\title{
Changement à la tête de «Swiss Medical Weekly»
}

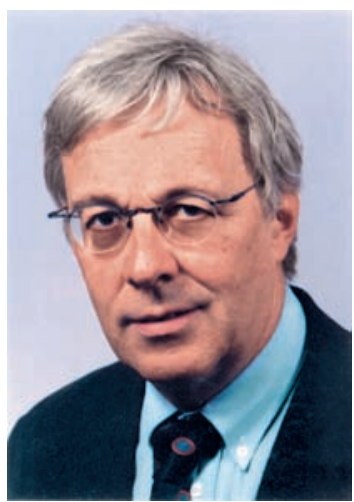

André P. Perruchoud
Notre petite Suisse a-t-elle besoin d'une revue de sciences médicales qui lui soit propre? On peut se poser la question à bon droit! Sur le plan international et particulièrement dans l'espace européen, il existe effectivement de nombreuses revues, bonnes pour la plupart et excellentes pour certaines, donnant des nouvelles de première importance dans les domaines les plus divers.

C'est exact, à deux exceptions près. La discipline «médecine interne» n'est dotée que d'un tout petit nombre de revues au top niveau, cachant derrière elles un vide plus ou moins béant. Et c'est ce vide que l'hebdomadaire «Swiss Medical Weekly» a l'intention de combler. Un deuxième point doit être mis en exergue: il concerne la relève académique. Le «Swiss Medical Weekly» tient à offrir à notre relève académique et futurs collègues un organe de publication utile et accessible pendant toute leur carrière.

En ma qualité de rédacteur en chef nouvellement élu, je dois naturellement me demander si nous satisfaisons à ces deux exigences. Ces dernières années, le «Swiss Medical Weekly»a subi de profondes modifications. Il est devenu un journal purement scientifique et le «Swiss Medical Forum» a été créé en parallèle pour la formation postgraduée et continue. Nous avons passé des langues nationales à l'anglais, professionnalisé et internationalisé la relecture, gagné enfin des scientifiques d'envergure, l'international advisory board. Avant tout, chaque travail est examiné en détail et analysé scientifiquement par les statisticiens mandatés par le SMW. Le nombre de consultations du site Internet s'élève à plus de 100000 par mois, dont 8000 directement de la Medline; les internautes accèdent au plein texte de SMW par un lien direct partant de Pubmed. Je félicite mon prédécesseur pour cette réussite et l'en remercie vivement.

Ces mesures, mais avant tout les manuscrits de qualité élevée et leurs excellents auteurs tout aussi bons, ont augmenté le facteur impact de 0,258 (2000) à 1,537 (2004). Ces deux dernières années, ce facteur a malheureusement de nouveau stagné. Nous devons donc accroître nos efforts, obtenir encore plus de travaux de qualité supérieure, aptes à être pris en considération et à être cités. Pour y par-venir, nous devons viser un facteur impact de 3,0. Tel est notre objectif pour ces quatre prochaines années; il est certes ambitieux mais pas irréalisable.

Il nous faut tout d'abord continuer dans la voie de l'amélioration et notamment accélérer le processus de relecture. En deuxième lieu, il nous faut gagner, pour la rédaction de revues scientifiques, plus d'auteurs reconnus sur le plan national et international. Troisièmement, la maison d'éditions doit mettre en œuvre la stratégie «open access» qu'elle a adoptée au sens de la déclaration de Berlin. Il s'agit, en l'occurrence, d'un pas courageux, souhaité par les universités mais difficile sur le plan financier. La science doit être ouvertement accessible!

Je consacrerai tout mon temps de rédacteur en chef à la poursuite de ces objectifs; ce n'est rien de particulier, c'est le point le plus important de mon contrat. Je peux entièrement compter sur le soutien et les connaissances de mes excellents collègues du comité éditorial. Le «Swiss Medical Weekly» a aussi besoin de l'aide extérieure: institutions académiques soucieuses de la relève suisse, sociétés de discipline médicale qui soutiennent le «Swiss Medical Weekly» pas seulement avec des mots mais aussi par des actes, jeunes universitaires qui cherchent l'accès à notre revue.

On aborde souvent une nouvelle tâche avec un souhait précis. Je suis convaincu que les sciences font progresser la médecine, mais que la médecine est soutenue par le front. Et ce front est constitué, en Suisse, par les lectrices et les lecteurs du Bulletin des médecins suisses. De vous, chères collègues et chers confrères, on attend des capacités pratiques, des connaissances théoriques et des compétences sociales, mais aussi une lecture critique et une mise en œuvre de la littérature professionnelle. Vous voyez où je veux en venir? Peut-être aurez-vous, vous aussi, envie de jeter un coup d'œil critique dans le «Swiss Medical Weekly».

Prof. André P. Perruchoud, rédacteur en chef du SMW 